\title{
Formación de comunidades políticas afines y disímiles en Twitter durante la campaña electoral a la alcaldía de Manizales en $2015^{\star}$
}

\author{
Luis Miguel López Londoño** \\ Recibido: 2018-10-02. Enviado a pares: 2018-10-15. \\ Aprobado por pares: 2018-11-29. Aceptado: 2018-12-15 \\ https://doi.org/10.22395/angr.v17n34a6
}

\begin{abstract}
Resumen
En el campo de la comunicación política, las redes sociales se han convertido en los últimos años en una herramienta indispensable en las campañas electorales. Además de abrir canales de comunicación y diálogo no disponibles en otros espacios mediáticos y públicos, estas plataformas promueven el surgimiento de comunidades en línea que generan conversaciones e intercambio de opiniones políticas. Sin embargo, la participación democrática en la era digital puede suponer la superación de ciertos obstáculos, pero también su profundización. Esta investigación aplicó un análisis de contenido a los mensajes publicados durante los últimos veinticinco días de campaña en las cuentas oficiales de Twitter de los cuatro aspirantes a la alcaldía de Manizales de 2015, con el objetivo de determinar si los seguidores de estas cuentas confrontaron sus puntos de vista con perspectivas disímiles o si los expresaron únicamente frente a usuarios políticamente afines. Los resultados hablan de una diferencia central entre las dos cuentas con mayor participación de los ciudadanos en cuanto a la formación de comunidades políticas: en solo una se propicia el encuentro entre voces políticamente opuestas, pero son voces que apelan al agravio, a la hostilidad y a la descalificación del otro como legítimo portador de una opinión. Pero las dinámicas de las dos cuentas plantean una misma consecuencia: la fragmentación de la esfera pública, la polarización y la disolución de la esencia de la democracia deliberativa.
\end{abstract}

Palabras clave: esfera pública; comunicación política; Twitter; interacción; polarización; fragmentación; comunidades políticas; democracia.

Este artículo ofrece algunos resultados del proyecto Agor@s y esfer@s emergentes: el Twitter como espacio de interacción en tiempos de campaña, investigación financiada por la Dirección de Investigaciones y Posgrados de la Universidad de Manizales y liderada por el autor de este texto, inscrito en la línea de lenguajes y narrativas del Grupo de Investigaciones de la Comunicación, adscrito a la Escuela de Comunicación Social y Periodismo de la Facultad de Ciencias Sociales y Humanas, de dicha universidad (Caldas, Colombia).

** Comunicador social-periodista y especialista en Opinión Pública y Marketing Político de la Pontificia Universidad Javeriana, magíster en Filosofía de la Universidad de Caldas, profesor asociado de la Escuela de Comunicación Social y Periodismo de la Facultad de Ciencias Sociales y Humanas de la Universidad de Manizales, profesor de Teorías de la Comunicación y Seminario de Investigación, asignaturas desde las cuales ha abordado el estudio de la opinión pública, la deliberación y la participación ciudadana en el contexto digital. Estudiante de segundo año de Doctorado en Communication Studies en Ohio University, Estados Unidos. Correo electrónico: Imlopez@umanizales.edu.co. Orcid: https://orcid.org/0000-0001-7542-4121 


\title{
Twitter formation of like-minded and dissimilar political communities during the 2015 mayoral campaign in Manizales
}

\begin{abstract}
In the field of political communication, social networks have become an indispensable tool for election campaigns in recent years. In addition to opening communication and dialogue channels that are not available in other media and public spaces, these platforms promote the emergence of online communities that spawn conversations and exchange of political opinions. However, democratic participation in the digital age can lead to overcome certain obstacles, but also to deepen them. A content analysis technique to the messages published during the last twenty-five days of the 2015 mayoral campaign in the official Twitter accounts of the four candidates for Manizales Mayor's Office, aimed at establishing if the followers of these accounts confronted their points of view with dissimilar perspectives, or if they expressed them only to users who were politically sympathetic to them. The results show a pivotal difference between the two accounts with the greatest participation of citizens regarding the formation of political communities: only one of facilitates the meeting between politically opposed voices, but they appeal to grievance, hostility and disqualification of the other as someone who has an opinion. Nevertheless, the dynamics of both accounts raise the same consequence: The fragmentation of the public sphere, the polarization and the dissolution of the essence of deliberative democracy.
\end{abstract}

Keyword: public sphere; political communication; Twitter; interaction; polarization; fragmentation; political communities; democracy.

\section{Formação de comunidades políticas afins e dissímeis no Twitter durante a campanha eleitoral à prefeitura de Manizales em 2015}

\begin{abstract}
Resumo
No campo da comunicação política, as redes sociais se converteram, nos últimos anos, em uma ferramenta indispensável nas campanhas eleitorais. Além de abrir canais de comunicação e diálogo indisponíveis em outros espaços midiáticos e públicos, essas plataformas promovem o surgimento de comunidades on-line que geram conversas e intercâmbio de opiniões políticas. No entanto, a participação democrática na era digital pode supor a superação de certos obstáculos, mas também seu aprofundamento. Esta pesquisa aplicou uma análise de conteúdo às mensagens publicadas durante os últimos 25 dias de campanha nas contas oficiais do Twitter dos quatro aspirantes à prefeitura de Manizales em 2015, com o objetivo de determinar se os seguidores desses perfis confrontaram seus pontos de vista com perspectivas dissímeis ou se os expressaram unicamente diante dos usuários politicamente afins. Os resultados apontam para uma diferença central entre as duas contas com maior participação dos cidadãos com respeito à formação de comunidades políticas: o encontro de vozes politicamente opostas é propiciado somente em uma, mas são vozes que apelam à queixa, à hostilidade e à desqualificação do outro como legítimo portador de uma opinião. Mas as dinâmicas das duas contam apresentam uma mesma consequência: a fragmentação da esfera pública, a polarização e a dissolução da essência da democracia deliberativa.

Palavras-chave: esfera pública; comunicação política; Twitter; interação; polarização; fragmentação; comunidades políticas; democracia.
\end{abstract}




\section{Introducción}

El 25 de octubre de 2015 se llevaron a cabo en el territorio colombiano las elecciones de gobernadores, alcaldes, diputados, concejales y ediles. En la ciudad de Manizales, capital del departamento de Caldas, cuatro candidatos aspiraron a ser elegidos como alcalde para el periodo 2016-2019. Adriana Gutiérrez recibió el aval del Centro Democrático, partido de extrema derecha y principal opositor al gobierno del otrora presidente de Colombia, Juan Manuel Santos. Octavio Cardona fue avalado por el Partido Liberal; Luis Fernando Acebedo por la Alianza Verde, con el apoyo de dos partidos de izquierda, el Polo Democrático y la Unión Patriótica; y Luis Roberto Rivas, quien anteriormente se había desempeñado como alcalde de la ciudad, recibió el apoyo de la Alianza por Manizales, una coalición de dirigentes liberales y conservadores. Al final de la jornada, Cardona se impuso con un total de 49.466 votos, equivalentes al 29,32\%. Luis Roberto Rivas obtuvo 44.255 votos (26,23\%), Adriana Gutiérrez, 43.013 (25,49\%) y Luis Fernando Acebedo, 18.881(11,19\%).

La radio, la televisión y la prensa, más vinculados con los poderes del Estado y del capital (Keane, 1997), han comprimido gradualmente los espacios para el cruce de mensajes entre candidatos y ciudadanos. Los procesos políticos se caracterizan por la escasa deliberación y la ausencia de voces diversas (Wilhelm, 2000)'. Pero la arquitectura de internet ha agrandado los espacios para que los ciudadanos manifiesten sus opiniones e intercambien una pluralidad de puntos de vista a través de la conversación pública (Blumler y Gurevitch, 2001; Papacharissi, 2002; Dahlgren, 2005; Friedland, Hove y Rojas, 2006). Las redes sociales ofrecen un modelo de producción y consumo de información que contrasta fuertemente con los procesos centralizados de distribución de contenidos de los medios de comunicación tradicionales (Conover, Gonçalves, Flammini y Menczer, 2012). Las campañas electorales las han sumado a sus estrategias y encontrado en ellas un innovador canal de contacto con simpatizantes y ciudadanos (Bode, Hanna, Yang y Shah, 2015). Estas plataformas son cada vez más populares para la comunicación política (Himelboim, McCreery y Smith, 2013) y son una herramienta para proveer información, coordinar actividades, atraer potenciales votantes y fomentar la discusión sobre los asuntos públicos (Feller, Kuhnert, Sprenger y Welpe, 2011; Hanna, Wells, Maurer, Shah, Friedland y Matthews, 2013).

Es precisamente el uso del Twitter durante la campaña a la alcaldía de Manizales el que ha motivado esta investigación, sustentada en el interés por describir y caracterizar la forma en que candidatos y seguidores interactuaron en esta red social en los últimos días de campaña. Propósito que se extiende a determinar, de manera más específica, si los segundos confrontan sus puntos de vista con perspectivas disímiles, o si, por el contrario, los expresan únicamente frente a usuarios afines políticamente. Para tal fin, se empleó el análisis de contenido con un enfoque mixto, para examinar de manera cuantitativa y cualitativa los mensajes publicados en esta red social. Si esta plataforma digital puede convertirse en un lugar para la reunión de voces y opiniones, es primordial entonces contribuir a la teoría sobre la esfera pública. Cuando los medios tradicionales

Todas las traducciones del inglés fueron realizadas por el autor de este artículo. 
han bloqueado y restringido el tránsito de las voces ciudadanas durante las campañas políticas, para concentrar su atención en las de los candidatos, es ineludible explorar los nuevos territorios en donde las primeras se hacen visibles y tienen eco. Estas dinámicas transforman las maneras en las que se comunica la política, los procesos democráticos y la participación ciudadana. La comunicación digital se encuentra en un proceso de expansión y está abriendo nuevas miradas y horizontes, por lo que es indispensable ampliar, desde un enfoque empírico, el estudio del uso de las redes sociales en los contextos electorales. En consecuencia, esta investigación se propuso responder al siguiente interrogante: ¿Cómo es la formación de comunidades políticas en Twitter durante los últimos veinticinco días de campaña en las cuentas oficiales de Twitter de los cuatro aspirantes a la alcaldía de Manizales de 2015?

Para cumplir con este propósito, primero se abordará el componente teórico que supone el estudio de las dinámicas comunicativas y participativas propiciadas por las redes y las nuevas tecnologías de la información. Luego se hará descripción del recorrido metodológico de la investigación, la presentación y el análisis de los resultados, y las conclusiones que de ellos se derivan.

\section{Nuevas redes de voces y opiniones}

Las nuevas tecnologías de la información han renovado la escenografía mediática y reconfigurado la función de los actores involucrados en los procesos de comunicación. Castells (2009) conceptualiza una nueva forma de sociedad, la sociedad red, en la que una geometría de redes locales y globales estructura las prácticas comunicativas e informativas en un espacio de interacción social. La cultura de la convergencia, señala Jenkins (2008), representa un cambio de paradigma, en el que los contenidos fluyen a través de canales mediáticos múltiples e interdependientes a los que se accede de distintos modos, y en donde productores y consumidores son impredecibles en su interacción. Las características de la reticularidad y la interactividad permiten el tránsito de un esquema de "uno a muchos" con receptores pasivos, a un modelo participativo de "muchos a muchos", en el que surge un receptor activo con la capacidad de producir contenidos (Scolari, 2008). De acuerdo a Dahlgren (2005), la relevancia del internet consiste especialmente en su capacidad para generar interacción cívica desde una comunicación horizontal. El proceso de interacción entre el usuario y el objeto mediático convierte al primero en coautor de la obra: elige qué elementos se muestran y qué rutas se siguen, generando una obra única (Manovich, 2005).

El número de actores implicados en la formación y expresión de la opinión pública ha cambiado. El internet ha "potenciado las dimensiones discursivas de una esfera pública hasta entonces gestionada por periodistas, encuestadores y políticos que acabaron por constituir un mismo entramado institucional (o con intereses compartidos)" (Sampedro y Resina, 2010, p.145). Ya no es la opinión pública medida y publicada, sino la opinión pública construida y compartida en tiempo real por nuevos protagonistas. Herramientas interactivas como chats, foros de debate, redes sociales y blogs contribuyen al diálogo 
político (Himelboim et al., 2013) y permiten que la actual sociedad de la opinión repose en la comunicación desmediatizada (Han, 2014). Las personas están explorando nuevas formas de ser ciudadanos y hacer política (Dahlgren, 2005). Una de ellas es la creación de comunidades en línea con creencias, identidades e ideologías compartidas (Bode et al., 2015).

El entramado técnico de la web permite la interactividad constante entre los usuarios, la expresión a gran escala de la opinión y el crecimiento de un hipertexto global (Friedland et al., 2006). Favorece la creación de macroesferas públicas en las que los ciudadanos generan conversaciones y "controversias con otros miembros de una remota ‘comunidad imaginaria" (Keane, 1997, p.68). Sin embargo, la misma tecnología que favorece la libre interacción entre ciudadanos distantes y de perspectivas diversas, concede a sus usuarios la opción de limitar y restringir el acceso a determinadas discusiones y fuentes de información (Himelboim et al., 2013). Si bien estas tecnologías logran reunir y agrupar a las personas, también suponen el riesgo de conducirlas en direcciones diferentes (Papacharissi, 2002).

Estas significativas transformaciones han impulsado un ejercicio de renovación y caracterización alrededor de la teoría sobre la esfera pública en un universo constituido por redes e interacciones virtuales. Esta tarea ha partido de la revisión del modelo liberal de esfera pública propuesto por Habermas (1981) y se ha orientado al análisis sobre la participación de los públicos en la discusión sobre los asuntos comunes, su impacto en la vida democrática y las posibilidades que ofrece la red como nuevo foro y arena para la comunicación y la deliberación política (Kim, Wyatt y Katz, 1999; Sunstein, 2001; Papacharissi, 2002; Dahlgren, 2005; Friedland et al., 2006; Dahlberg, 2007b; Waisbord, 2015).

El creciente uso del Twitter por parte del público, los partidos y los candidatos ha incrementado considerablemente la investigación en torno al papel de esta red social en las campañas electorales (Jungherr, 2016). Algunos estudios establecen la relación entre el uso del Twitter y su efecto en las preferencias de los votantes (Kruikemeier, 2014; Raynauld y Greenberg, 2014). Otros han caracterizado el uso del Twitter por parte de los candidatos a distintos cargos de elección popular (Castells, 2009; Aparaschivei, 2011; Borondo, Morales, Losada y Benito, 2012; Vergeer y Hermans, 2013; Zamora y Zurutuza, 2014; Jungherr, Schoen y Jürgens, 2016).

En el entorno regional y local, Barredo, Arcila y Arroyave (2015) examinaron la influencia de las redes sociales en la decisión de voto de los ecuatorianos. Slimovich (2016) evaluó los discursos políticos que los candidatos a la presidencia de Argentina en 2011 publicaron en sus cuentas de Twitter. García-Perdomo (2017) estudió la forma en que cien periodistas y sus seguidores en Twitter encuadraron la campaña presidencial de 2014 en Colombia. Desde la teoría de la identidad y el personalismo político, Cifuentes y Pino (2018) analizaron los tuits de los congresistas del Centro Democrático para identificar la presencia de agendas temáticas comunes.

Un amplio rango de estudios ha explorado la disposición de los públicos para participar de la discusión pública. En algunas ocasiones, depende de variables como 
la heterogeneidad de la red, la experticia política y el consumo previo de noticias (Moy y Gastil, 2006); de la expresión de la opinión pública en situaciones de presión social (Scheufele, 1999) o de las preferencias políticas y los temas que se discuten (Feller et al., 2011). Distintas investigaciones han explorado la conformación de comunidades en línea, afines o disímiles ideológicamente (Stromer-Galley, 2003; Eveland y Hively, 2009; Conover et al., 2012; Himelboim et al., 2013; Bode et al., 2015), mientras que otras han concentrado en las consecuencias que para la democracia tiene la formación de dichas comunidades (Price, Cappella y Nir, 2002; Sunstein; 2002, Dahlberg, 2007a; Hanna et al., 2013; Morales, Borondo, Losada y Benito, 2015).

\section{Metodología}

La red social Twitter está constituida por una serie de aplicaciones que facilitan la comunicación en diferentes formatos: texto, imágenes, audio, video y enlaces con otros sitios web. Su naturaleza consiste en compartir información, en tiempo real y desde cualquier lugar, a través de mensajes de un máximo de 140 caracteres conocidos como tuits. Estos contenidos son recibidos por un grupo de ciudadanos (denominados "seguidores") aprobado por el titular de la cuenta y pueden ser retransmitidos a nuevas audiencias por medio de la opción "Retuitear". Estos mensajes se conocen como retuits.

El clic en la opción "Responder" abre un espacio en blanco en el que el ciudadano puede replicar y contestar, bajo la denominación de respuesta o comentario, los mensajes tuiteados o retuiteados. Este mecanismo permite observar los comportamientos comunicativos de los usuarios y la emergencia de diferentes comunidades discursivas (Bode et al., 2015), algunas de ellas organizadas alrededor de palabras o frases precedidas del símbolo numeral (\#) conocidas como hashtags. La estructura de estas conversaciones es la de un discurso que genera otros discursos que dialogan solamente con el primero. Pero la lógica de muchos textos que responden a uno solo se altera y suscita una multiplicidad de textos que remiten unos a otros pero que no siempre guardan conexión con el que los generó (Raimondo, Reviglio y Divisni, 2015). El usuario de Twitter puede interactuar con el titular de la cuenta, con otros seguidores o con ambos.

Precisamente, esta investigación parte de la definición y el alcance del concepto interacción. Según Dahlgren (2005), esta ocurre en dos circunstancias. Una es el resultado del encuentro entre el ciudadano y el medio digital, es decir, la forma en que el primero interpreta y usa la información. La otra es el encuentro y el "cara a cara en línea" entre los ciudadanos a partir de sus conversaciones, las cuales varían en extensión y en participantes. La conversación política se encuentra en el núcleo de la democracia deliberativa, entendida esta última como aquel proceso en el que los ciudadanos, de manera libre y voluntaria, comparten información sobre las cuestiones públicas, construyen opiniones y participan en los procesos políticos (Kim et al., 1999).

Este estudio empleó el análisis de contenido para describir y clasificar temáticamente los mensajes publicados durante los últimos días de campaña. Como una de las técnicas 
de investigación más importantes en las ciencias sociales, el análisis de contenido examina "los datos dentro de un contexto específico en vista de los significados que alguien -un grupo o una cultura- les atribuye" (Krippendorff, 1989, p. 403). Para dicha tarea, el análisis de los datos debe realizarse con respecto al contexto en el que surgen (Krippendorff, 1990). En este caso, se analizaron las informaciones publicadas en las cuentas de Twitter de los cuatro postulantes al cargo de alcalde de Manizales en las elecciones de 2015. Por lo tanto, fue necesario el estudio del contexto político local y nacional, imprescindible para comprender el fondo y el significado de muchos de los mensajes publicados en esta red social. Desde las posibilidades que ofrece esta plataforma para la interacción comunicativa entre los usuarios, el análisis de contenido es la herramienta más conveniente para analizar, tanto los componentes y características de los mensajes, como la reciprocidad entre sus emisores (Wilhelm, 2000).

Desde este marco metodológico, la investigación se orientó con un enfoque mixto. De acuerdo a la actividad de candidatos y seguidores en cada una de las cuentas, se realizó un análisis cuantitativo de los mensajes publicados desde el primero hasta el veinticinco de octubre, incluyendo el día de la elección. Aunque los candidatos habían emprendido sus actividades proselitistas desde finales del mes de julio, diferentes estudios muestran que el volumen de mensajes tiende a crecer al final de la campaña (Jungherr, 2016), momento en el que los ciudadanos empiezan a perfilar sus decisiones de voto y en el que los equipos de campaña intensifican sus tareas persuasivas y comunicativas. De igual forma, se aplicó un análisis cualitativo. Se dividieron los mensajes en unidades temáticas y luego a cada una de estas se le asignaron categorías y subcategorías (para lograr mayor especificidad) de acuerdo a su contenido y al contexto en el que surgieron.

Se tuvo acceso a la información haciendo parte de los seguidores en la cuenta oficial de Twitter de cada aspirante. Se recopilaron, de forma manual 3481 mensajes, equivalentes a 1324 tuits, 1045 retuits y 1112 respuestas generadas por sus seguidores.

Para la codificación de estos mensajes se diseñó un instructivo de aplicación con las siguientes categorías: fecha, hora, tipo de mensaje (tuit, retuit o respuesta), contenido, menciones (@), tendencias (\#), total de retuiteos y respuestas de cada mensaje, y número de usuarios que publicaron respuestas. Con respecto al contenido y a la intención del emisor, estos mensajes se clasificaron en las categorías temáticas de la tabla 1.

Adicionalmente, desde los objetivos planteados en la investigación, de la categoría ataques, críticas y acusaciones se derivaron subcategorías. Es importante señalar que la mayoría de las categorías elegidas para el análisis de los mensajes son el resultado de un ejercicio deductivo. Primero se hizo una revisión de la literatura publicada por diferentes autores sobre el uso de redes sociales en campaña para determinar cuáles categorías habían sido utilizadas; luego, se procedió a identificarlas en los mensajes recopilados. Sin embargo, también se procedió a categorizar de manera inductiva los mensajes que, por su contenido y naturaleza específica del contexto en el que se surgieron, no correspondían con categorías empleadas en estudios previos (tabla 2). 
Tabla 1. Categorías temáticas de los tuits, retuits y respuestas publicados en las cuentas de los cuatro candidatos

\begin{tabular}{ll}
\hline \multicolumn{1}{c}{ Tuits/retuits } & \multicolumn{1}{c}{ Respuestas } \\
\hline Ataques, críticas y acusaciones & Ataques, críticas y acusaciones \\
\hline Publicidad política & Publicidad política \\
\hline Propuestas & Preguntas de usuario a candidato \\
\hline Actividades de campaña & Preguntas de usuario a usuario \\
\hline Mensajes religiosos & Propuesta de conversación \\
\hline Mensajes de gratitud & Repetición del mensaje \\
\hline Otros & Otros \\
\hline
\end{tabular}

Fuente: elaboración propia

Tabla 2. Subcategorías temáticas de la categoría ataques, críticas y acusaciones ${ }^{2}$

\begin{tabular}{l}
\hline \multicolumn{1}{c}{ Ataques, críticas y acusaciones } \\
\hline A Álvaro Uribe y al uribismo \\
\hline Defensa de Álvaro Uribe y del uribismo \\
\hline A otro usuario \\
\hline A Juan Manuel Santos y al santismo \\
\hline A las FARC y a los acuerdos de paz \\
\hline Al (a los) candidato (s) \\
\hline A la dirigencia política en general \\
\hline Al Fiscal General de la Nación \\
\hline A los partidos y los dirigentes de izquierda \\
\hline Otros \\
\hline
\end{tabular}

Para asegurar la clasificación de los contenidos sin apreciaciones subjetivas, este ejercicio fue desarrollado por dos codificadores, cada uno de los cuales realizó dos rondas de análisis. Luego se procedió a comparar los resultados y resolver los desacuerdos, ya que en muchos casos fue necesario analizar tuits anteriores para determinar la categoría del mensaje. 2 Los términos uribismo y santismo se utilizan en el argot político para referirse a la ideología y al legado de Álvaro Uribe
y Juan Manuel Santos, respectivamente. Uribistas y santistas son los partidarios de dicha ideología. 


\section{Resultados}

Antes de avanzar en la interpretación de los resultados, valga aclarar que con excepción del Centro Democrático y algunos líderes conservadores, todos los movimientos y partidos políticos que participaron en esta elección han apoyado los esfuerzos del gobierno por encontrar una salida negociada al conflicto con las Fuerzas Armadas Revolucionarias de Colombia (FARC), en el marco de las negociaciones emprendidas en Cuba desde agosto de $2012^{3}$. Este hecho profundizó la división que desde 2010 ya había empezado a incubarse entre el expresidente y máximo líder de este naciente partido, Álvaro Uribe Vélez, y Juan Manuel Santos, mandatario de los colombianos. La decisión de este último de restablecer las relaciones diplomáticas con el gobierno de Hugo Chávez y la designación de algunos ministros y funcionarios de alto nivel, significaron el preludio de la ruptura de la amistad política entre estos dos dirigentes. Desde ese momento, la enemistad y las acusaciones entre ambas corrientes sentaron las bases para la fuerte división y polarización que actualmente copa la agenda política nacional. Este contexto es importante para entender la interacción entre usuarios en la plataforma@Adrigutijara presentada anteriormente.

Las cuentas de Adriana Gutiérrez (@Adrigutijara)y Octavio Cardona (@jcardonaleon) fueron las más activas durante los últimos veinticinco días de campaña, al concentrar el 63 \% de la actividad, la primera con el 33 \% y la segunda con el 30 \%. Luis Roberto Rivas (@LuisRobertoAlc) representó el 24 \% y Luis Fernando Acebedo (@luisacebedo) el 13\%. Estos dos últimos candidatos, contrario a Gutiérrez y Cardona, otorgaron más importancia a los mensajes provenientes de otras cuentas (retuits) que a los mensajes originados desde sus propias cuentas (tuits). Los seguidores de@Adrigutijaray@jcardonaleon generaron muchas más respuestas ante los mensajes publicados que los seguidores de las otras plataformas (figura 1).

En cuanto al número de seguidores aceptados por el titular de cada cuenta,@Adrigutijara contaba el primero de octubre con 1531, cifra que se elevó a 1760 al cierre del día de la votación. En el mismo lapso de tiempo, @jcardonaleon pasó de 1142 seguidores a 1264; @luisacebedo,de 772 a 897; y @LuisRobertoAlc, de 766 a 884. Sin embargo,como se observa en la figura 2, la cifra de quienes dieron clic en la aplicación "Responder" es significativamente inferior al número total de seguidores, lo que significa que estos están más interesados en recibir información que en contestarla.

\footnotetext{
Finalizada la fase de negociación, el gobierno convocó a un plebiscito, realizado el 2 de octubre de 2016, con el fin de que los ciudadanos aprobaran los acuerdos. La campaña por el No, liderada por el Centro Democrático, obtuvo el 50,2 \% de los votos y derrotó a la campaña por el Sí, que alcanzó el 49,7 \%. Sin embargo, y con el fin de proteger los acuerdos, el gobierno acudió al Congreso de la República para que estos fueran aprobados. Para el momento en que se escribió este texto, se encontraban en fase de implementación.
} 


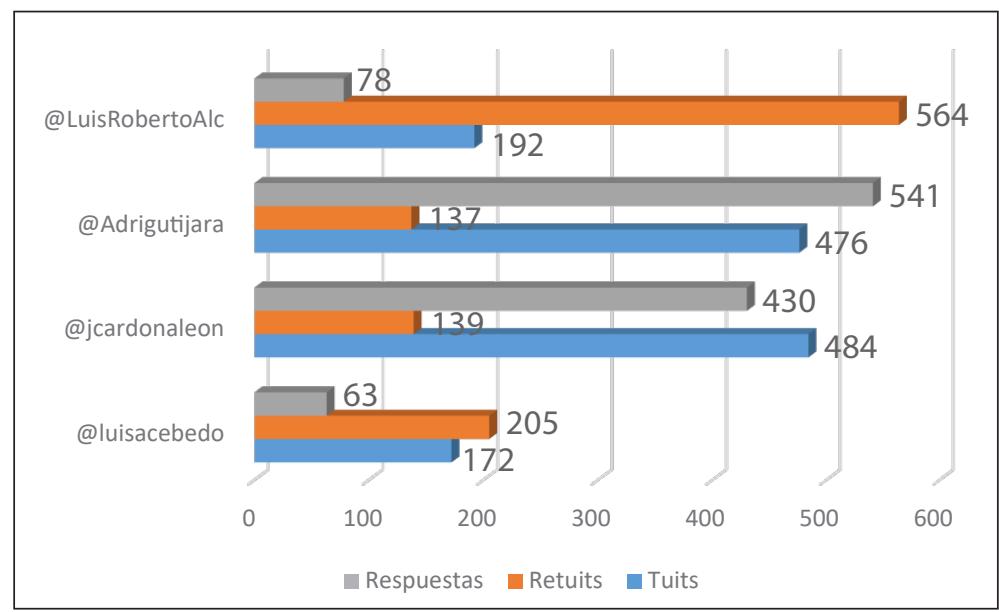

Figura 1. Mensajes publicados en las cuentas de los candidatos.

Fuente: elaboración propia

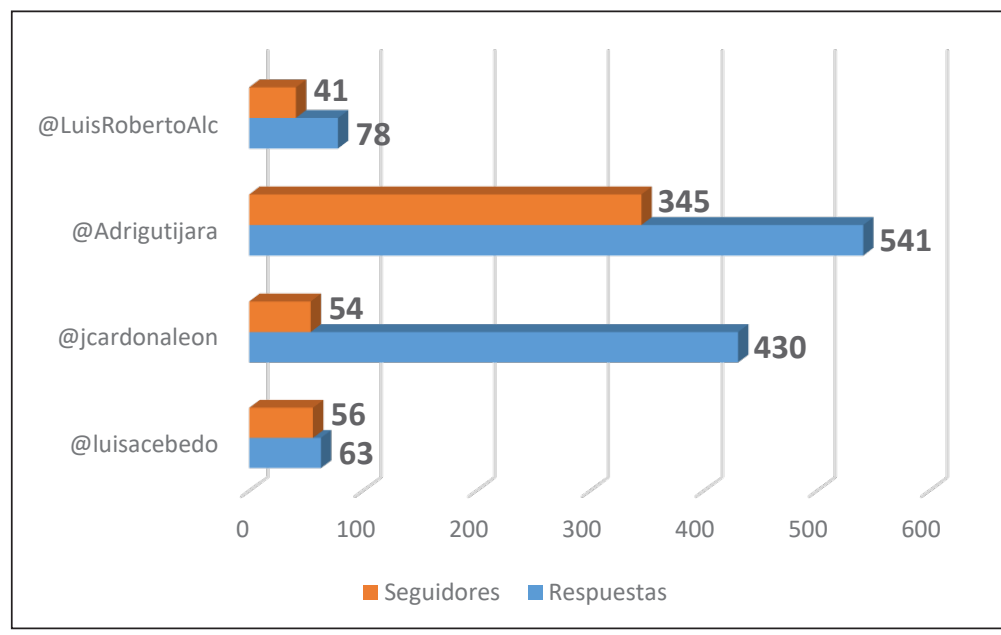

Figura 2. Número de seguidores que publicaron respuestas en las cuentas de los cuatro candidatos.

Fuente: elaboración propia

La interacción entre los seguidores de las dos cuentas con mayor número de respuestas constituye el hallazgo más destacado. De las 1112 respuestas publicadas por los seguidores en las cuentas de los cuatro candidatos, @Adrigutijara y @jcardonaleon concentraron la mayoría de la actividad, con el 48,6\% y el 38,7 \% respectivamente. Cifras muy distantes a las de @LuisRobertoAlc, con el 7\%, y @luisacebedo con el 5,7 \%. Por tal motivo, se eligieron las dos primeras cuentas como objeto de análisis. La identificación de las categorías con más comentarios en las cuentas de Cardona y Gutiérrez refleja que una sola categoría reúne la mayoría del porcentaje (tabla 3). 
Tabla 3. Categorías temáticas de las respuestas publicadas en las cuentas de Adriana Gutiérrez y Octavio Cardona

\begin{tabular}{lcclccc}
\hline @jcardonaleon & \multicolumn{7}{c}{$@$ @Adrigutijara } \\
\hline Categoría & $\#$ & $\%$ & Categoría & $\#$ & $\%$ \\
\hline Publicidad política & 375 & 87,2 & Ataques, críticas y acusaciones & 402 & 74,3 \\
\hline Preguntas de usuario a candidato & 20 & 4,7 & Publicidad política & 108 & 20 \\
\hline Ataques, críticas y acusaciones & 16 & 3,7 & Preguntas de usuario a candidato & 21 & 3,9 \\
\hline Otros & 19 & 4,4 & Otros & 10 & 1,8 \\
\hline Total & 430 & 100 & Total & 541 & 100 \\
\hline
\end{tabular}

Fuente: elaboración propia

De las 430 respuestas publicadas en @jcardonaleon, 375 de ellas, que equivalen al $87,2 \%$, corresponden a la clasificación publicidad política. Los mensajes incluidos en esta categoría aluden a las capacidades y cualidades del candidato, a sus bondades y valores como persona, a la originalidad de sus propuestas, a los tuits de los usuarios informando el candidato por el que votarán, frases de apoyo y cualquier otro contenido con fines propagandísticos y persuasivos. Estas respuestas fueron publicadas solamente por 34 seguidores diferentes y expresan de manera abierta su simpatía y afinidad con Cardona. Su intención es publicitar al aspirante y proyectar la imagen de un hombre virtuoso y capacitado. Los seguidores interactúan con los mensajes de otros usuarios y con el mensaje original del candidato.

Por otra parte, del total de respuestas publicadas en@jcardonaleon, únicamente siete (de seis usuarios diferentes), equivalentes al 1,6\%, corresponden a tuiteros que, de acuerdo al contenido e intención del mensaje, no comparten la ideología de Cardona. Estos comentarios corresponden a la categoría ataques, críticas y acusaciones. Estos mensajes, principalmente, cuestionan las campañas, las propuestas y los perfiles morales de los candidatos. En este caso, dudan de la transparencia y financiación de su campaña, de sus prácticas clientelistas y de su pasado como funcionario público. Exigen vehementemente explicaciones y descalifican a Cardona. De estas siete respuestas solo una fue replicada por parte del mismo candidato, nunca por sus seguidores, quienes no manifestaron interés en sostener encuentros en línea con posiciones contrarias.

Lo que ocurre en@Adrigutijara es completamente diferente. De los 541 comentarios publicados en la cuenta de la candidata del Centro Democrático, por parte de 345 seguidores diferentes, 402 (74,3\%) correspondieron a la categoría ataques, críticas y acusaciones. Además de cuestionar las campañas y los aspirantes a la alcaldía, en estos mensajes se ataca, con palabras hostiles y provocadoras, la postura ideológica de los seguidores que participan en la conversación (aunque las críticas y acusaciones se dirigen también, en una escala significativamente menor, a medios de comunicación y políticas públicas de administraciones locales o nacionales del momento). La intención de los 292 seguidores 
que participaron en este tipo de conversaciones es alinearse entre los dos bandos ideológicos enfrentados en esta plataforma: los defensores del legado de Álvaro Uribe y los simpatizantes del presidente Juan Manuel Santos.

Se destaca que, de esas 402 respuestas transmitidas, 352, correspondientes al 87,5 $\%$, se derivaron únicamente de seis mensajes. Un tuit que generó 83 comentarios y otros dos retuits que recibieron 134 y 55 respuestas, publicados por dos importantes dirigentes del Centro Democrático, tuvieron como tema el enfrentamiento entre los dos bloques políticos mencionados anteriormente. La razón fue el anuncio de la Fiscalía General de la Nación de compulsar copias a la Corte Suprema de Justicia para que se investigara a expresidente Álvaro Uribe, por su presunta participación, cuando era Gobernador de Antioquia, en la masacre cometida por grupos paramilitares en El Aro, municipio de Ituango, en octubre de 1997. Uno de esos retuits iba acompañado de una imagen del rostro sonriente del dirigente y el hashtag \#LoOueEsConUribeEsConmigo, tendencia que invitaba a sus simpatizantes a rodear a su líder y a defender su causa, ante un gobierno que él mismo acusaba de haber emprendido una persecución judicial en su contra. El tuit incluía otro hashtag: \#SoyUribistaNoTerrorista. Este se convirtió en tendencia en Twitter una vez que Roy Barreras, congresista del Partido de la U, acusara a los integrantes del Centro Democrático se ser los nuevos terroristas, debido a su negativa de apoyar las conversaciones que en ese momento buscaban una salida pacífica al conflicto entre el gobierno y las FARC.

Los otros tres retuits, con 47, 17 y 16 réplicas, respectivamente, corresponden a la categoría publicidad política. En este caso se invitaba "al uribista a votar uribista" y a reiterar que Gutiérrez haría respetar a Manizales como su alcaldesa. Las conversaciones que se desprendieron de estos tres mensajes mantuvieron como fondo político el mismo enfrentamiento señalado anteriormente.

Hay tres factores importantes por destacar en estos seis mensajes. El primero tiene que ver con la estructura de la conversación. De un mensaje original se empiezan a desprender otros que dialogan con él; pero también surgen comentarios de otros comentarios, que en algunos casos se alejan de ese mensaje inicial. Estas conversaciones se asemejan a un tejido o red de anuncios interconectados a manera de comunidades dialógicas.

El segundo, con las temáticas que se desprenden del enfrentamiento entre los dos bloques ya mencionados. Uno de ellos defiende el legado uribista, muestra su rechazo a los acuerdos de paz y ve con preocupación la llegada del "modelo castro-chavista" a Colombia; acusa al Fiscal de ese entonces, Eduardo Montealegre, es hostil con los dirigentes y los partidos de izquierda y desconoce las cualidades de Juan Manuel Santos como presidente. El otro bloque defiende la gestión de este último y su iniciativa de paz, reitera frecuentemente los escándalos de corrupción del gobierno cuando Uribe fue mandatario, denuncia la cercanía de este con los paramilitares y el narcotráfico y rememora el episodio de los falsos positivos. Algunos mensajes inculpan a estas dos corrientes por constituirse en actores irreconciliables y reflejar lo más común y habitual de la política nacional. 
Hay ataques que desbordan estos asuntos, pero no se desprenden completamente de ellos. La capacidad mental y racional de algunos ciudadanos es puesta en duda, pero depende de su cercanía ideológica con alguna de estas dos corrientes. La candidata Gutiérrez es agraviada por su pertenencia al Centro Democrático, pero es también alabada por ser incondicional y fiel a los principios del partido. Los seguidores se enfrentan entre ellos con lenguajes provocadores, señalando su simpatía hacia algún grupo armado al margen de la ley: si defiende los acuerdos de paz, es guerrillero, si no lo hace, es paramilitar.

El tercer factor es el encuentro en línea de ideologías opuestas, de ciudadanos que comparten opiniones y puntos de vista contrarios. Contrario a lo que ocurre en @ jcardonaleon, la cuenta de Gutiérrez es el escenario para la reunión de perspectivas antagónicas. Desde las dinámicas comunicativas presentes en estos tejidos de respuestas, es posible identificar dos líneas políticas que se enfrentan y desafían, que se provocan y descalifican, con lenguaje hostil y ofensivo. El eje del conflicto y la disputa son las desavenencias entre Álvaro Uribe y Juan Manuel Santos. La agenda local y de ciudad estuvo subordinada a esta finalidad.

\section{Discusión}

Las diferentes investigaciones sobre la participación política y la deliberación de los públicos han abierto dos frentes de análisis: los del encuentro en línea entre ciudadanos con la misma ideología y con ideologías opuestas. El primero se refiere a la decisión que toman los internautas de integrar comunidades de su misma identidad y pertenencia política, el segundo, a la disposición de exponerse a posturas contrarias y con las que no se está de acuerdo (Stromer-Galley, 2003; Dahlberg, 2007a). Ambos escenarios plantean, por un lado, unas consecuencias, algunas deseables otras no deseables. Por el otro, la importancia de la interacción cívica y la discusión en el contexto de la democracia deliberativa (Dahlgren, 2005), ya que este ejercicio amplía el repertorio de argumentos y fomenta la comprensión de múltiples puntos de vista (Price et al., 2002).

El primer campo de análisis plantea que, en el mundo de la política online, los ciudadanos y los "centros de reunión" tienen más probabilidades de enlazarse con aquellos que son similares (Wilhelm, 2000; Friedland et al., 2006; Himelboim et al., 2013). Morales et al. (2015) y Price et al. (2002) sostienen que experiencias recientes han demostrado que los usuarios políticamente más activos interactúan principalmente con sus partidarios, y dejan poco espacio para el debate real y las interacciones con ideologías opuestas. En algunos sitios web y chats, los públicos se mezclan escuchando únicamente las perspectivas que desean escuchar y evadiendo la exposición a otras alternativas (Stromer-Galley, 2003). Es lo que Eveland y Hively (2009) denominan "discusión segura".

Al respecto, Dahlberg (2007a) explica la evaluación de algunos investigadores en torno a las discusiones en red: el debate en estos grupos se mueve hacia la homogeneidad ideológica (entendida como el grado el que los mensajes individuales se adhieren a 
una cierta afiliación política). Es un fenómeno conocido como "homofilia" (Huckfeldt y Sprague, 1995, citados por Wilhelm, 2000), que explica la tendencia de los individuos a asociarse con sus similares. Aunque el internet supone la presencia de una enorme diversidad de identidades y puntos de vista, mucha de la interacción online implica la reunión de individuos afines, quienes evitan debatir con ideas diferentes y desvían las posiciones contrarias. "Las razones pueden ser múltiples: desinterés, prejuicios, percepción selectiva, y preferencia por permanecer en una zona de comodidad con el consumo de información e ideas que se ajustan a intereses existentes" (Waisbord, 2015, p. 15).

Estas dinámicas se presentan en la interacción generada a partir de las respuestas publicadas en@jcardonaleon por parte de sus seguidores: no hay visibilidad para opiniones contrarias. La comunidad de tuiteros que participan con comentarios es homogénea en su composición, ya que estos últimos responden al interés principal de promocionar al candidato, pero no de contradecir o cuestionar determinada afirmación. Se publica más en función de la propaganda positiva que desde la disposición a generar un debate desde orillas opuestas.

Las consecuencias y riesgos varían. Según Dahlberg (2007a), se limita la posibilidad de comprender el punto de vista de los otros, lo que a su vez puede incrementar la hostilidad o generar incluso violencia. Se reducen los beneficios que derivan de exponerse frente a puntos de vista opuestos y problemas inadvertidos. Los temores al malestar social pueden inhibir o anular la expresión de puntos de vista disidentes, incluso cuando las personas privadamente no están de acuerdo (Price, 2002). El resultado de que algunas personas deliberen solo con grupos afines es la polarización: no solamente sus visiones se refuerzan, sino que pueden ir más allá y desplazarse, incluso, a puntos más extremos (Sunstein, 2002; Stromer-Galley, 2003). A la polarización entre los públicos le sigue el fracaso de la esfera pública y la desestabilización social (Dahlberg, 2007a).

La discusión entre individuos con la misma identidad política e intereses lleva a la fragmentación de la esfera pública (Dahlberg, 2007a), traducida, según Sunstein (2002), en la formación de "enclaves deliberativos", aquellos grupos en los que los ciudadanos, al oír ecos de sus propias voces, terminan reforzando más aún sus posturas. El internet, reitera, está haciendo posible que las personas diseñan sus propios "paquetes de comunicaciones" en los que filtran las cuestiones problemáticas y las voces contrarias. La democracia puede convertirse, según Waisbord (2015), en un archipiélago de múltiples diferencias con débiles interacciones comunicativas, con el consecuente riesgo de la disolución de los lazos sociales.

Ahora bien, las respuestas en la cuenta de Gutiérrez generaron una dinámica opuesta. Este es el segundo campo de análisis. Algunas investigaciones aseguran que los participantes en línea buscan la deliberación con actores que sostienen puntos de vista marcadamente diferentes. Según estos estudios, el internet está siendo utilizado por muchas personas para el encuentro con la diferencia, que normalmente no encuentran en la vida cotidiana. El desacuerdo es vital porque, según Price et al. (2002), obliga a una 
consideración más cuidadosa de los puntos de vista desafiantes, lo que lleva a exigir, a quienes deliberan, la construcción de opiniones mejor razonadas. Esta es la esencia de la democracia deliberativa: la conversación entre aquellos que expresan puntos de vista disímiles (Kim et al., 1999), lo que a su vez implica encontrar en internet la posibilidad de ampliar la esfera pública Dahlberg (2007a).

Los análisis de Price et al. (2002) y Moy y Gastil (2006) señalan que la probabilidad de encontrar desacuerdo y confrontación se eleva considerablemente a medida que las redes de conversación se hacen más grandes en tamaño y más heterogéneas en composición. Precisamente, la cuenta@Adrigutijara fue la que sumó un mayor número de seguidores y de ciudadanos que publicaron comentarios o respuestas. Destacan estos autores las ventajas de encontrarse con el desacuerdo en la conversación política: contribuye a la construcción de argumentos más razonados y a comprender las razones de por qué otros esgrimen razones contrarias. La investigación de Stromer-Galley (2003) da cuenta del interés de los usuarios por interactuar con perspectivas opuestas: la posibilidad de acceder a juicios desconocidos, de percibir el clima de opinión y de profundizar la perspectiva sobre algún asunto público. Estas conversaciones son valiosas en cuanto a que los participantes emergen de ellas con una comprensión más clara de las razones y valores subyacentes a los argumentas contrarios.

No obstante, la deliberación que se despliega en @Adrigutijara entre dos bloques antagónicos de seguidores no corresponde propiamente al ideal de esfera pública, en el que el debate responde a los principios de la tolerancia y el reconocimiento del otro. Si bien la deliberación requiere que sus participantes estén abiertos al conflicto político (Moy y Gastil, 2006), uno de los resultados posibles de estos diversos espacios de discusión es que las personas terminan gastando más tiempo discutiendo y atacándose entre sí que involucrándose en la deliberación racional (Stromer-Galley, 2003). Internet se concibe como un medio y un territorio para la lucha y el conflicto político (Dahlberg, 2007b), en donde los contenidos son negativos e irónicos frente a los candidatos y partidos (Jungherr, 2016).

Aunque la plataforma de Gutiérrez es la de más actividad por parte de sus seguidores, una mayor participación en la discusión política no resulta automáticamente en una discusión que promueva ideales democráticos (Papacharissi, 2002). Si bien la cuenta de la candidata del Centro Democrático alienta y estimula el debate, este se desarrolla con un lenguaje belicoso e incitador, en el que el comentario opositor es descalificado y reducido en su validez y significado. Es lo que Eveland y Hively (2009) denominan "discusión peligrosa". En algunas ocasiones, el internet se convierte en el escenario para la disputa y la hostilidad, para el agravio y la intolerancia (Dahlgren, 2005; Friedland et al., 2006; Bode et al., 2015). Incluso, muchas veces la conversación no se despliega estrictamente en un ambiente político (Himelboim et al., 2013). La discusión entre los seguidores de @ Adrigutijara se desvía del enfrentamiento uribistas-santistas para concentrarse en las capacidades o "incapacidades" mentales de quienes participan en ella. 
La presencia de las voces discordantes en@Adrigutijara es vista como amenaza, no como oportunidad. No hubo otra razón distinta al enfrentamiento político entre Álvaro Uribe y Juan Manuel Santos para que estas voces se encontraran. Con un volumen de desprecio y efervescencia, ofrecieron en la red social Twitter una fiel radiografía de lo que es hoy la división política a nivel nacional, la que a su vez coincide con otros estudios. GarcíaPerdomo (2017) destacó la fascinación de los seguidores de las cuentas de Twitter de los periodistas analizados, por los mensajes que encuadran una confrontación directa entre los candidatos como característica principal para entender las elecciones y la campaña política. Cifuentes y Pino (2018) señalaron que los congresistas del Centro Democrático, desde la identificación de dos antagonistas principales, las FARC y el presidente Juan Manuel Santos, utilizaron el Twitter para atacar a opositores del partido.

Por fuera de estos dos territorios de análisis, merece especial atención la baja participación de los seguidores en las conversaciones y en la divulgación de mensajes. En la cuenta de Cardona, las 430 respuestas fueron publicadas por solo 54 seguidores, pero 345 de estas corresponden únicamente a seis tuiteros. Esta es una de las dificultades de la democracia deliberativa (Dahlgren, 2005). Aquellos que tienen acceso a internet no necesariamente persiguen la discusión y la interacción (Himelboim et al., 2013), dominada con frecuencia por muy pocos usuarios (Papacharissi, 2002). Es una constante en las diferentes investigaciones sobre el uso del Twitter como vehículo para la conversación política: muchos contribuyendo muy poco y pocos contribuyendo mucho (Jungherr, 2016). Aunque el nivel de participación en @Adrigutijara es mucho mayor que el de Cardona, todavía es significativamente bajo. En la discusión surgida entre simpatizantes y detractores de Uribe y Santos, participaron 292 usuarios, un número muy alejado de los 1531 seguidores que acumulaba Gutiérrez el primero de octubre y de los 1760 que sumaba al cierre del día de la votación.

De acuerdo a los resultados anteriormente mencionados y analizados, la publicación de mensajes a) de un mismo contenido político en@jcardonaleon, sin presencia de voces disímiles que estimulen el debate y la conversación cívica, b) de diferentes contenidos en @Adrigutijara, pero con una concentrada dosis de agresividad y hostilidad y c) el poco interés de los seguidores de los candidatos de interactuar en conversaciones con los demás, invitan a examinar lo planteado por Dahlgren (2005): internet ofrece una variedad de posibilidades para los encuentros cívicos, pero no ofrece todavía las soluciones para algunas fisuras de la democracia.

\section{Conclusiones}

El objetivo de esta investigación se concentró en analizar y caracterizar la interacción entre candidatos y ciudadanos en la red social Twitter, en el contexto de las elecciones a la alcaldía de Manizales del año 2015. Finalidad que se extendió a la tarea de determinar si aspirantes y tuiteros desplegaron conversaciones con actores contrarios y con posturas diferentes o si, por el contrario, los diálogos no superaron el marco de las comunidades afines y semejantes. 
Los principales resultados señalan que las dos cuentas con mayor número de respuestas por parte de sus seguidores,@Adrigutijara y@jcardonaleon, presentan casos contrarios. En la primera hubo encuentro entre opiniones diferentes, lo que constituye la esencia misma de la democracia deliberativa y la posibilidad de ampliar y enriquecer la esfera pública. Sin embargo, esa esencia se diluye ante la prevalencia de los mensajes hostiles, el agravio y la opinión intolerante. La presencia de voces discordantes se entiende como un peligro y una contingencia, pero no como la oportunidad para fortalecer los valores mismos del debate político: la racionalidad, el respeto y el reconocimiento del otro. En la segunda cuenta, la presencia única de perspectivas amigas también contribuye a la disolución del sentido de la discusión pública. La interacción entre ciudadanos de la misma línea ideológica, más que fortalecer el espíritu democrático del intercambio de ideas, supone la fragmentación de la esfera pública, la radicalización y la división de la sociedad en bloques polarizados e irreconciliables.

Si bien las herramientas interactivas contribuyen al encuentro de las voces ciudadanas y reconfiguran las dinámicas de los actores involucrados en el ejercicio de la deliberación, aún es muy apresurado afirmar que las nuevas tecnologías han superado viejos obstáculos. Este estudio ofrece una fotografía del momento político de la región y el país, pero eventos electorales posteriores, bajo contextos cambiantes, pueden reflejar comportamientos e interacciones comunicativas diferentes. Futuras miradas al mundo político de la web podrían determinar si la presencia o ausencia de interacción entre visiones opuestas están impactando las dinámicas democráticas, y si estos tipos de interacción resuelven algunos problemas ya existentes o los profundizan.

\section{Referencias}

Aparaschivei, P. (2011). The use of Nueva media in electoral campaigns: Analysis on the use of blogs, Facebook, Twitter and YouTube in the 2009 Romanian presidential campaign. Journal of Media Research, 4(2), 39-60.

Barredo, D., Arcila, C., y Arroyave, J. (2015). Influence of Social Networks in the Decision to Vote: An Exploratory Survey on the Ecuadorian Electorate. International Journal of E-Politics, 6(4), 15-34. doi: 10.4018/IJEP.2015100102

Blumler, J. y Gurevitch, M. (2001). The New Media and Our Political Communication Discontents: Democratizing Cyberspace. Information, Communication y Society 4(1), 1-13. doi: 10.1080/713768514

Bode, L., Hanna, A., Yang, J., y Shah, D. (2015). Candidate networks, citizen clusters, and political expression: Strategic Hashtag use in the 2010 midterms. The Annals of the American Academy of Political and Social Science, 659(1), 149-165. doi:10.1177/0002716214563923

Borondo, J., Morales, A., Losada, J. y Benito, R. (2012). Characterizing and modeling an electoral campaign in the context of Twitter: 2011 Spanish Presidential election as a case study. Chaos, 22(2). doi: $10.1063 / 1.4729139$

Castells, M. (2009). Comunicación y poder. Madrid: Alianza.

Cifuentes, C. y Pino, J. (2018). Conmigo o contra mí: análisis de la concordancia y estrategias temáticas del Centro Democrático en Twitter. Palabra Clave, 21 (3), 885-916. doi: 10.5294/pacla.2018.21.3.10 
Conover, M., Gonçalves, B., Flammini, A., y Menczer, F. (2012). Partisan asymmetries in online political activity. EPJ Data Science, 1(1), 1-19. doi:10.1140/epjds6

Dahlberg, L. (2007). Rethinking the fragmentation of the cyberpublic: from consensus to contestation. Nueva Media y Society, 9(5), 827-847. doi: 10.1177/1461444807081228

Dahlberg, L. (2007). The Internet, deliberative democracy, and power: Radicalizing the public sphere. International Journal of Media and Cultural Politics, 3(1), 47-64. doi: 10.1386/macp.3.1.47/1

Dahlgren, P. (2005). The Internet, Public Spheres, and Political Communication: Dispersion and Deliberation. Political Communication, 22(2), 147-162. doi: 0.1080/10584600590933160

Eveland, W. y Hively, M. (2009). Political discussion frequency, network size, and "heterogeneity" of discussion as predictors of political knowledge and participation. Journal of Communication, 59(2), 205-224. doi:10.1111/j.1460-2466.2009.0141

Feller, A., Kuhnert, M., Sprenger, T. y Welpe, I. (2011). Divided they tweet: The network structure of political microbloggers and discussion topics. En N. Nicolov, J. G. Shanahan, L. Adamic, R. BaezaYates y S. Counts (Eds.), ICWSM 2011 : Proceedings of the 5 th International AAAI Conference on Weblogs and Social Media (pp. 474-477). Menlo Park: Association for the Advancement of Artificial Intelligence (AAAI). Recuperado de https://www.aaai.org/ocs/index.php/ICWSM/ICWSM1 1/paper/view/2759

Fredland, L., Hove, T. y Rojas, H. (2006). The networked public sphere. Javnost-The Public, 13(4), 5-26. doi: $10.1080 / 13183222.2006 .11008922$

García-Perdomo, V. (2017). Between peace and hate: Framing the 2014 Colombian presidential election on Twitter. Cuadernos.info, 41, 57-70. https://doi.org/10.7764/cdi.41.1241

Habermas, J. (1981). Historia y crítica de la opinión pública: la transformación estructural de la vida pública. Barcelona: Gustavo Gili.

Han, B. (2014). En el enjambre. Barcelona: Herder.

Hanna, A., Wells, C., Maurer, P., Shah, D., Friedland, L. y Matthews, J. (2013). Partisan alignments and political polarization online: A computational approach to understanding the French and US presidential elections. En I. Weber, A. M. Popescu y M. Pennacchiotti (Eds.), PLEAD 2013: Proceedings of the Politics, Elections, and Data Workshop (pp. 15-21). Nueva York: ACM. Recuperado de http://alexhanna.com/static/pdf/Hanna_etal.PLEAD2013.pdf

Himelboim, I., McCreery, S. y Smith, M. (2013). Birds of a feather tweet together: Integrating network and content analyses to examine cross-ideology exposure on Twitter. Journal of Computer-Mediated Communication, 18(2), 40-60. doi:10.1111/jcc4.12001

Jenkins, H. (2008). Convergence culture: la cultura de la convergencia de los medios de comunicación. Barcelona: Paidós.

Jungherr, A. (2016). Twitter use in election campaigns: A systematic literature review. Journal of Information Technology and Politics, 13(1), 72-91. doi: 10.1080/19331681.2015.1132401

Jungherr, A., Schoen, H., y Jürgens, P. (2016). The mediation of politics through Twitter: An analysis of messages posted during the campaign for the German federal election 2013. Journal of ComputerMediated Communication, 21(1), 50-68. doi:10.1111/jcc4.12143

Kim, J., Wyatt, R., y Katz, E. (1999). News, Talk, Opinion, Participation: The Part Played by Conversation in Deliberative Democracy. Political Communication, 16(4), 361-385. doi:10.1080/105846099198541 
Keane, J. (1997). Transformaciones estructurales de la esfera pública. Estudios Sociológicos, 43, 47-77.

Krippendorff, K. (1989). Content analysis. En E. Barnouw, G. Gerbner, W. Schramm, T. L. Worth y L. Gross (Eds.), International encyclopedia of communication (pp. 403-407). Nueva York: Oxford University Press. Recuperado de http://repository.upenn.edu/asc_papers/226

Krippendorff, K. (1990). Metodología de análisis de contenido: teoría y práctica. Barcelona: Paidós.

Kruikemeier, S. (2014). How political candidates use Twitter and the impact on votes. Computers in Human Behavior, 34, 131-139. doi:10.1016/j.chb.2014.01.025

Manovich, L. (2005). El lenguaje de los nuevos medios de comunicación: la imagen en la era digital. Barcelona: Paidós.

Morales, A.J., Borondo, J., Losada, J.C. y Benito, R.M. (2015). Measuring political polarization: Twitter shows the two sides of Venezuela. Chaos, 25(3), 1-9. doi:10.1063/1.4913758

Moy, P. y Gastil, J. (2006). Predicting Deliberative Conversation: The Impact of Discussion Networks, Media Use, and Political Cognitions. Political Communication, 23, 443-460. doi: 10.1080/10584600600977003

Papacharissi, Z. (2002). The virtual sphere: the internet as a public sphere. Nueva Media and Society, 4(1), 9-27. doi: 10.1177/14614440222226244

Price, V., Cappella, J. y Nir, L. (2002). Does Disagreement Contribute to More Deliberative Opinion? Political Communication, 19(1), 95-112. doi:10.1080/105846002317246506

Raimondo, N., Reviglio, M. y Divisni, R. (2015). Esfera pública y redes sociales en Internet ¿Qué es lo nuevo en Facebook? Revista Mediterránea de Comunicación, 7(1), 211-229. doi: 10.14198/ MEDCOM2016.7.1.12

Raynauld, V.y Greenberg, J. (2014). Tweet, click, vote: Twitter and the 2010 Ottawa municipal election. Journal of Information Technology y Politics, 11(4), 412-434. doi:10.1080/19331681.2014.935840

Sampedro, V., y Resina, J. (2010). Opinión pública y democracia deliberativa en la Sociedad Red. Ayer, 80 (4), 139-162. Recuperado de http://www.ciberdemocracia.net/articulos/Ayer80SampedroyResina.pdf

Scheufele, D. A. (1999). Deliberation or dispute? An exploratory study examining dimensions of public opinion expression. International Journal of Public Opinion Research, 1 1, 25-58. doi: 10.1093/ijpor/1 1.1.25

Scolari, C. (2008). Hipermediaciones: elementos para una teoría de la comunicación digital interactiva. Barcelona: Gedisa.

Slimovich, A. (2016). Política y redes sociales en Argentina: el caso de los candidatos presidenciales de 2011 en Twitter. Signo y Pensamiento, 68, 86-100. doi:10.11144/Javeriana.syp35-68.prsa

Stromer-Galley, J. (2003). Diversity of Political Conversation on the Internet: Users' Perspectives. Journal of Computer-mediated Communication 8(3). doi: 10.1111/j.1083-101.2003.tb00215.x

Sunstein, C. (2001). Republic.com. Princeton: Princeton University Press. Recuperado de http://jolt.law. harvard.edu/articles/pdf/v14/14HarvJLTech753.pdf

Sunstein, C. (2002). The Law of Group Polarization. Journal Of Political Philosophy, 10(2), 175-195. doi:10.1111/1467-9760.00148

Vergeer, M. y Hermans, L. (2013). Campaigning on Twitter: Microblogging and online social networking as campaign tools in the 2010 general elections in the Netherlands. Journal of Computer-Mediated Communication, 18(4), 399-419. doi:10.1111/jcc4.12023 
Waisbord, S. (2015). Diversidad, diferencia, tolerancia. Revisando utopías democráticas a la luz de la comunicación digital. En A. Rojas (Presidencia), Convergencias comunicativas. Mutaciones de la cultura y del poder. Conferencia Magistral del XV Encuentro Latinoamericano de Facultades de Comunicación Social, Medellín, Colombia.

Wilhelm, A. G. (2000). Democracy in the digital age: Challenges to political life in cyberspace. Nueva York: Routledge.

Zamora, R. y Zurutuza, C. (2014). Campaigning on Twitter: Towards the "Personal Style"" campaign to activate the political engagement during the 2011 spanish general elections. Communication $y$ Society, 27(1), 83-106. 